\title{
PLASTIC STRESS-STRAIN FIELDS AND BLUNTING EFFECTS DURING LARGE DEFORMATIONS
}

\author{
H. Q. LIU and G. C. LI \\ Institute of Mechanics, Academia Sinica, Beijing 100080, P.R.C.
}

\begin{abstract}
Plastic stress-strain fields of two types of steel specimens loaded to large deformations are studied. Computational results demonstrate that, owing to the fact that the hardening exponent of the material varies as strain enlarges and the blunting of the crack tip, the well known HRR stress field in the plane strain model can only hold for the stage of a small plastic strain. Plastic dilatancy is shown to have substantial effects on strain distributions and biunting. To justify the constitutive equations used for analysis and to check the precision of computations, the load-deflection of a three-point bend beam and the load-elongation of an axisymmetric bar notched by a V-shaped cut were tested and recorded. The computed curves are in good accordance with experimental data.
\end{abstract}

\section{INTRODUCTION}

IT IS NOW AN accepted fact that the stress intensity factor in linear elasticity can characterize the singularity condition near a crack tip only when the plasticity is limited within a small-scalc yiclding zone. Using a total deformation theory of plasticity, in conjunction with power-law hardening materials, Hutchinson [1] and Rice and Rosengren [2] presented the distributions of stress occurring at the tip of a crack for both plane stress and plane strain. This singular behaviour of stress under plastic conditions is known as the HRR field. A full understanding of its usefulness and limitation is absolutely necessary for appropriate application. Recently, Aoki et al. [3] shed light on this important subject by claiming that the field near a crack tip can be divided into four characteristic fields (in sequence the $K$ field, HRR field, blunted crack tip field and the damaged region around the crack tip). Using a plastic dilatational constitutive model, Li et al. [4] also came to the conclusion that there is an obvious drop in the stress distribution near the crack tip. Based on these facts, we should avoid applying the IIRR field to the damage zone.

In this paper we intend to study the plastic stress-strain fields of two types of specimens loaded to large deformations. The effects of plastic dilatancy under large deformations and of blunting near either crack tip or notch tip on the stress-strain fields are examined. The quantitative extent of employing the HRR field for a plane-strain crack is estimated with the background of a low carbon low alloy steel.

The updated Lagrangian formulation developed by McMeeking and Rice [5] is used for finite element analysis, in conjunction with the plastic dilatational constitutive equations proposed by $\mathrm{Li}$ and Howard [6] and recently re-examined by $\mathrm{Li}$ et al. [4]. The plastic tangent moduli of the material were determined by a previous test and justified by some computer simulations as done by Xia et al. [7]. The macroscopic responses of a three-point bend beam and of an axisymmetric bar notched by a V-shaped cut are given and compared with the computational results. Two numerical examples are in good accord with the experimental curves.

\section{CONSTITUTIVE MODEL FOR DUCTILE MATERIALS}

In view of the plastic dilatancy existing in ductile materials loaded to large deformations, the elastic-plastic dilatational constitutive equations proposed by $\mathrm{Li}$ and Howard [6] are adopted. The formulation is not dependent on a certain plastic potential nor on the so-called convexity and normality rule; the basis of derivation is an equivalent transformation between the expressions used for the rate of dissipated plastic work (see the re-examination work of Li et al. [4]).

According to this theory, the total plastic deformation rate $D_{j}^{i(p)}$ is composed of two parts:

$$
D_{j}^{i(p)}=\frac{9}{4 E_{l e}^{(p)}} \frac{S_{j}^{i} S_{k}^{l}}{\sigma_{e}^{2}} \frac{\mathscr{D} \tau_{l}^{k}}{\mathscr{D} t}+\delta_{j}^{i} \frac{1}{3 E_{l m}^{(p)}} \frac{\mathscr{D} \tau_{k}^{k}}{\mathscr{D} t} .
$$


The first term on the right-hand side of eq. (1) represents the deviatoric part, whilst the second term is the corresponding volumetric contribution. $\tau_{l}^{k}$ is the Kirchhoff stress tensor. $E_{t e}^{(p)}$ and $E_{m}^{(p)}$ are the plastic tangent moduli, which are defined as

$$
E_{l c}^{(p)}=\frac{\mathscr{D} \sigma_{c} / \mathscr{D} t}{D_{c}^{(p)}} \quad E_{t m}^{(p)}=\frac{\mathscr{D} \tau_{k}^{k} / \mathscr{D} t}{D_{l}^{(p)}}
$$

$\mathscr{D} / \mathscr{D} t$ denotes the Jaumann rate with respect to the generalized time $t$. It is easy to check that

$$
\begin{aligned}
\sigma_{o} & =\left(\frac{3}{2} S_{j}^{i} S_{i}^{j}\right)^{1 / 2} \quad \text { (equivalent stress) } \\
S_{j}^{i} & =\tau_{j}^{i}-\delta_{j}^{i}\left(\frac{1}{3} \tau_{k}^{k}\right) \quad \text { (deviatoric stress) } \\
D_{e}^{(p)} & =\left(\frac{2}{3} d_{j}^{i(p)} d_{i}^{(p)}\right)^{1, ?} \quad \text { (plastic equivalent deformation rate) } \\
d_{i}^{j(p)} & =D_{i}^{j(p)}-\delta_{i}^{j}\left(\frac{1}{3} D_{i}^{(p)}\right) \quad \text { (plastic deviatoric deformation rate) } \\
\sigma_{m} & =\frac{1}{3} \tau_{k}^{k} \quad \text { (mean stress) } \\
D_{m}^{(p)} & =\frac{1}{3} D_{i}^{(p)} \quad \text { (plastic mean deformation). }
\end{aligned}
$$

Therefore, $E_{i c}^{(p)}$ and $E_{t m}^{(p)}$ are respectively the tangent moduli along the curve of equivalent stress $\sigma_{e}$ versus plastic equivalent strain $\varepsilon_{v}^{(p)}$ and the curve of mean stress $\sigma_{m}$ versus plastic mean strain $\varepsilon_{m}^{(p)}$. Here

$$
\begin{aligned}
& \varepsilon_{e}^{(p)}=\int_{0}^{t} D_{e}^{(p)} \mathrm{d} t \\
& \varepsilon_{m}^{(p)}=\int_{0}^{t} D_{m}^{(p)} \mathrm{d} t .
\end{aligned}
$$

If no damage occurs and $E_{m}^{(p)} \rightarrow \infty$, then eq. (1) reduces to the incompressible plastic deformation rate, which can be described by the Prandtl-Reuss theory. In this case, loading surfaces have a convex shape similar to the one traced by the von Mises yield function. Then the convexity and normality rule holds and it is well known that as a consequence

$$
\frac{1}{E_{t c}^{(p)}}=\frac{1}{E_{t}}-\frac{1}{E}
$$

Here, $E_{t}=\mathrm{d} \sigma / \mathrm{d} \epsilon$ is the tangent modulus on a uniaxial stress-strain $(\sigma-\epsilon)$ curve. It should be noticed that eq. (5) is true only under the assumption of plastic incompressibility and uniform stress condition in the testing bars. After necking occurs (stress distribution is non-uniform) or when $E_{m m}^{(p)}$ becomes finite (plastic incompressibility assumption fails) and if ductile damage prevails (strain hardening may change to strain softening), then the situation is much more complicated. Both microstructural study and an incorporation of experimental records and computer simulations are needed for the determination of these constitutive parameters.

Assuming that the total deformation rate is composed of an elastic part following Hooke's law and a plastic component as given in eq. (1), eventually we obtain

$$
\begin{aligned}
D_{j}^{i} & =D_{j}^{i(e)}+D_{j}^{i(p)} \\
& =\frac{1}{E}\left[(1+v) \frac{\mathscr{D} \tau_{j}^{i}}{\mathscr{D} t}-v \delta_{j}^{i} \frac{\mathscr{D} \tau_{k}^{k}}{\mathscr{D} t}\right]+\frac{9}{4 E_{l e}^{(p)}} \frac{S_{j}^{i} S_{k}^{l}}{\sigma_{l}^{2}} \frac{\mathscr{D} \tau_{l}^{k}}{\mathscr{D} t}+\delta_{j}^{i} \frac{1}{3 E_{l m}^{(p)}} \frac{\mathscr{D} \tau_{k}^{k}}{\mathscr{D} t}
\end{aligned}
$$

with its inverse form as

$$
\frac{\mathscr{D} \tau^{i j}}{\mathscr{D} t}=\frac{E}{(1+v)}\left[\frac{1}{2}\left(g^{i k} g^{j l}+g^{i l} g^{j k}\right)+g^{i j} g^{k l} \frac{v-E / 3 E_{t m}^{(p)}}{1-2 v+E / E_{l m}^{(p)}}-\frac{3}{2 \sigma_{e}^{2}} \frac{E}{E_{l e}^{(p)}} \frac{S^{i j} S^{k l}}{\frac{2}{1}(1+v)+E / E_{l e}^{(p)}}\right] D_{k l} .
$$

\section{STRESS-STRAIN FIELDS AND BLUNTING UNDER LARGE DEFORMATIONS}

Based on the updated Lagrangian formulation [5], two types of specimens were computed, using the computational procedure explained by $\mathrm{Li}$ and Howard [6]. One example is a three-point 
bend beam in plane strain loading. The beam has a span $S=96 \mathrm{~mm}$ with its height $W=24 \mathrm{~mm}$ and a pre-existing crack length $a=13.1 \mathrm{~mm}$. The other is an axisymmetric bar of gross diameter $\phi=16 \mathrm{~mm}$ notched axisymmetrically by a V-shaped cut into net diameter $\phi_{0}=10 \mathrm{~mm}$. The geometrical dimensions, together with their finite element discretizations, are shown in Fig. 1. The half span of the three-point bend beam includes 755 triangular constant strain elements with 408 nodes, while a quarter of the axisymmetric bar uses 956 elements with 509 nodes.

The material parameters used for computations were taken from a series of tests reported by Xia et al. [7]. There, in order to estimate the plastic dilatation occurring in the ductile material loaded to large deformations, experimental observations were made not only on smooth cylinders but also on some round bars notched axisymmetrically by U-shaped cuts of different local radii, so that different values of triaxial stress-volumetric plastic strain can be obtained in the centre zone.

Since the local strains in the specimens may be far beyond necking, and substantial plastic dilatation is found in testings, the two tangent moduli in eq. (2) can no longer be determined through simple tension tests. So far, the only method is to resort to a computer simulation technique, which is virtually a 'trial and error' method. Varying the values of the tangent moduli fed into the computer, one tries to gain computed responses that fit the experimental results as closely as possible.

Adding the elastic contributions to the strains in eq. (4), we can obtain the total equivalent strains and mean strain. Figure $2 \mathrm{a}$ and $\mathrm{b}$ show respectively the equivalent stress-strain curve and the mean stress-strain curve, obtained through a computer simulation of the load-elongation results recorded in the tests done by Xia et al. [7] on a low carbon low alloy steel. Using the curve in Fig. 2a, we obtain the data listed in Table 1 for $E / E_{t e}^{(p)}$ versus $\varepsilon_{e}$. In Fig. 2b, the straight line represents the elastic part of volumetric change marked with $(e)$, whilst the plastic dilatation is shown by an additional portion of $(p)$. Based on this figure, we take $E / E_{l m}^{(p)}=1.1$ throughout our computations for the sake of simplicity.

It should be emphasized that the values of the moduli listed above are associated with an implicit size scale parameter, since these moduli are determined through computer simulation using the finite element method. It is a well known fact that the magnitudes of local stress and strain
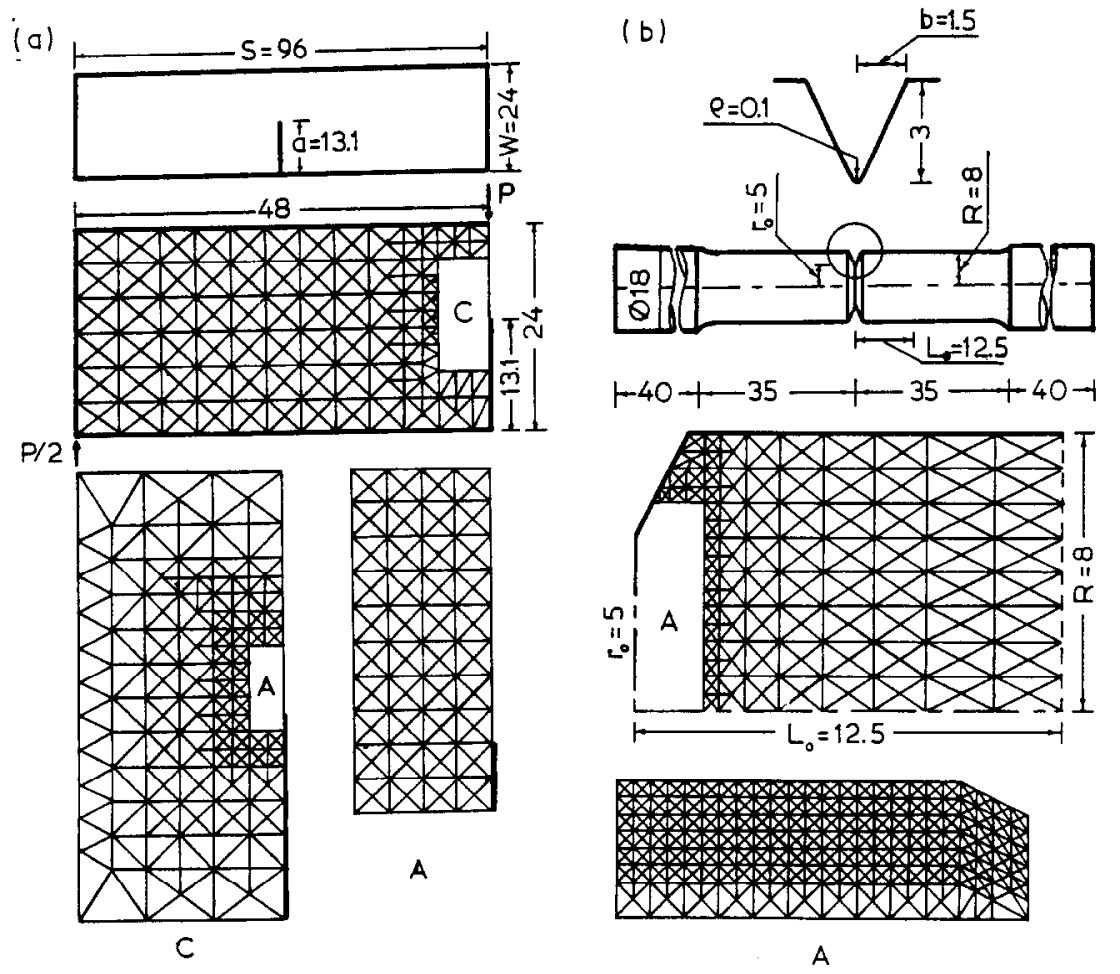

Fig. 1. Geometrical dimensions and finite element mesh (length in $\mathrm{mm}$ ). (a) Three-point bend beam. (b) Axisymmetric round bar. 

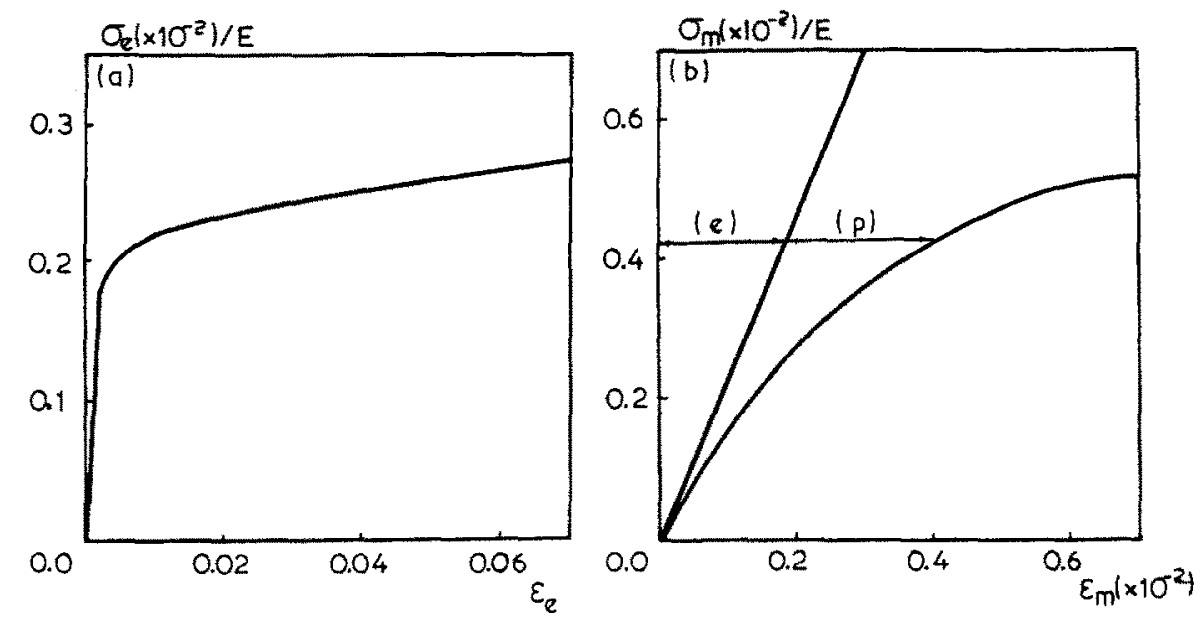

Fig. 2. The stress-strain curves of a low carbon low alloy steel. (a) Equivalent stress strain. (b) Mean stress-strain.

and the computed load elongation curves of the axisymmetric bars all depend on the size of the elements chosen. Therefore we need to implement a 'consistency' principle in choosing the size scale for the elements within the most sensitive area where stress and strain are concentrated. Owing to this requirement, we use a $250 \times 250 \mu \mathrm{m}$ square and subdivide it into four equal triangles as shown in the mesh parts termed $A$ in Fig. 1, to make it consistent with the smallest mesh size used previously by Xia et al. [7]. Another important point is that the mesh size should not be too small, since the determination of the constitutive parameters is based on a 'homogenized' estimation. The actual microstructure of the steel is spheroidite with hard-phase inclusions of about $4 \mu \mathrm{m}$ on average. Around these inclusions, primary voids may initiate. Secondary voids are also seen under higher stress-strain loading. Therefore each element should include enough numbers of the characteristic size of actual heterogeneity in order to smear out the local discontinuity. Finally, the mesh size should be able to represent local concentration of stress-strain. Rousselier [8] used a mesh size of $500 \mu \mathrm{m}$. Our determination was based on a general balance and consideration of all the points stated here.

Figure 3 shows the spread of the plastic region in: (a) the ligament beyond the crack tip (c.t.) in the three-point bend beam, and (b) a quarter of the axisymmetric bar notched by a V-shaped cut. In the figure, $\Delta$ denotes the deflection at the middle of the beam and $\Delta L$ is the elongation of the bar within a length scale of $L_{0}$.

Figure 4 demonstrates the blunting feature at different loading stages. The crack tip is lowered in Fig. 4a as crack tip blunting increases; $\mathrm{d} X$ and $\mathrm{d} Y$ are respectively the horizontal and vertical distances measured from the initial crack tip position. The crack tip opening displacement, CTOD, is determined by the turning point of two tangential lines. The crack tip opening angle, CTOA, depends on the inclined angle of the tangential line at the tip. In accordance with the sequence of $\Delta / W=0.026,0.030,0.046$ and 0.053 , we have $C T O D=0.046,0.065,0.105$ and $0.140 \mathrm{~mm}$ with $\mathrm{CTOA}=10^{\circ} 25^{\prime}, 15^{\circ} 9^{\prime}, 23^{\circ} 38^{\prime}$ and $27^{\circ} 30^{\prime}$. Figure $4 \mathrm{~b}$ indicates that the value of CTOA accelerates after $\Delta / W=0.01$, and it results in obvious blunting. Figure $4 \mathrm{c}$ shows the change of the peripheral contour of the notched bar with respect to its initial position. The configuration of the notch tip constitutes an inclined angle called the notch tip angle, NTA. In Fig. 4d, the NTA speeds up quickly but slows down later. This is contrary behaviour when compared with that of the crack tip.

Table 1. $\boldsymbol{E} / \boldsymbol{E}_{t e}^{(p)}$ versus $\varepsilon_{e}$

\begin{tabular}{lccccc}
\multicolumn{7}{c}{ Table 1. $E / E_{t e}^{(p)}$ versus $\varepsilon_{e}$} \\
\hline$\varepsilon_{e}^{(p)}$ & 0.002 & 0.004 & 0.015 & 0.030 & 0.040 \\
$E_{\left(E_{i e}^{(p)}\right.}$ & 1000 & 1000 & 1000 & 84 & 102 \\
$\varepsilon_{e}^{(p)}$ & 0.050 & 0.065 & 0.080 & 0.120 & 0.200 \\
$E / E_{t e}^{(p)}$ & 143 & 183 & 257 & 476 & 500 \\
\hline
\end{tabular}




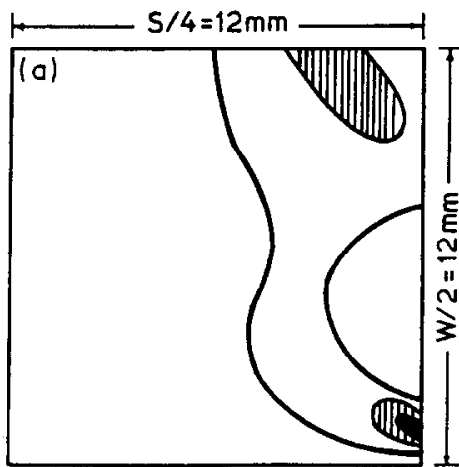

$\Delta / W=0.004$

$\square \Delta / W=0.009$

$\Delta / W=0.026$

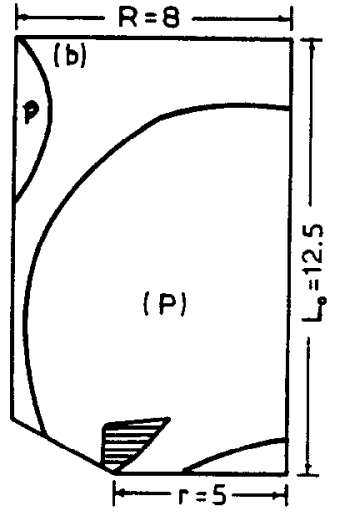

口 $\Delta L \Omega_{0}=0.0017$

(P) $\Delta L /=0.0053$

Fig. 3. The development of plastic area. (a) Three-point bend beam. (b) Axisymmetric bar.

In view of Figs 3 and 4, we can conclude that local blunting is based on a wide spread of plastic yielding. Blunting brings about not only concentration of the equivalent stress and strain but also a high amount of the mean stress and strain near the tips. Damage (or softening) occurs in the material used with a void volume fraction around $2 \%$ [7], which is tantamount to a mean strain of 0.007 . Therefore, the amount of mean strain caused by blunting naturally leads to initial damage around the tips. Figures 5 and 6 show the distributions of the stresses and strains near the tips and support the above viewpoint, in which the stresses are normalized by the yield stress $\sigma_{y}$.
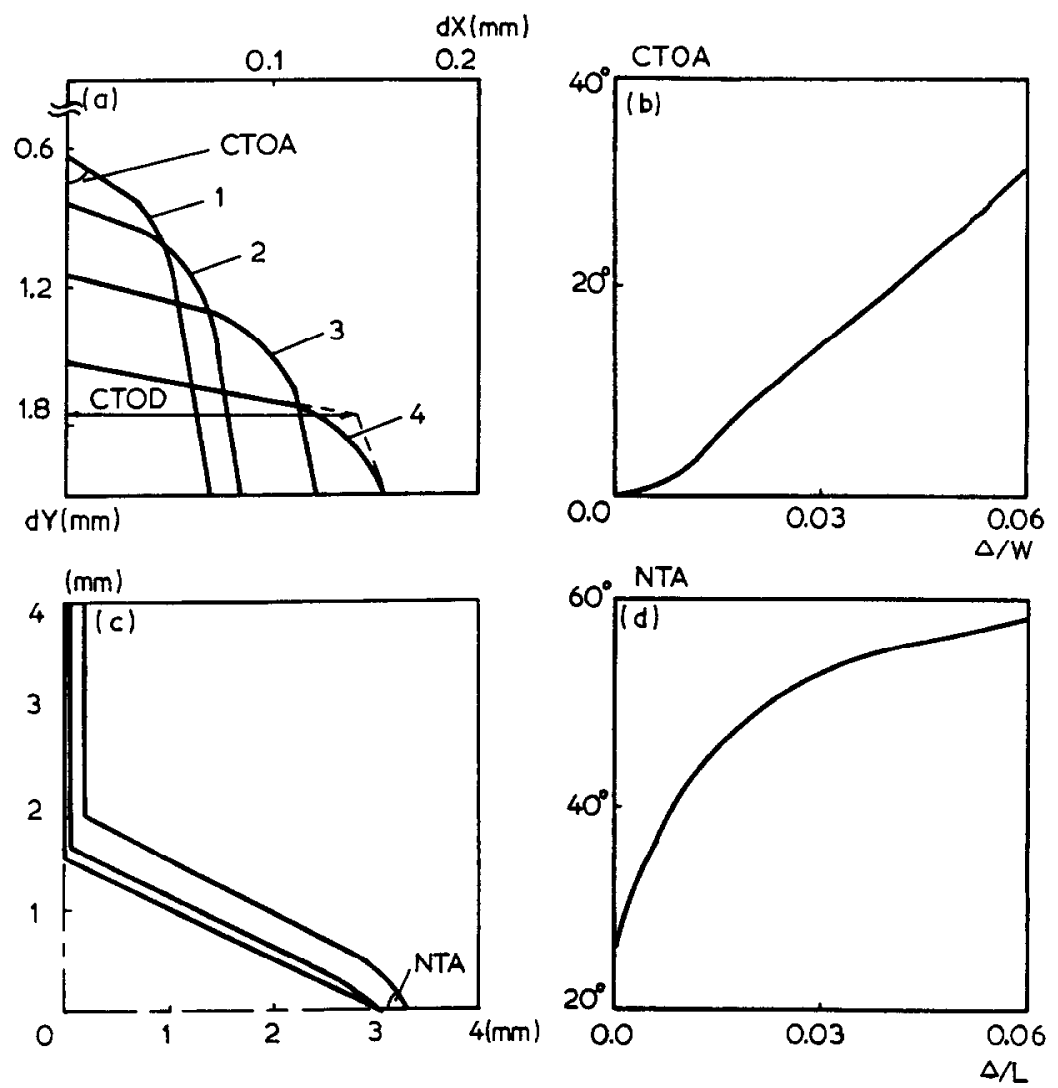

Fig. 4. The blunting features of the crack tip (a, b) and of the notch tip (c, d). 

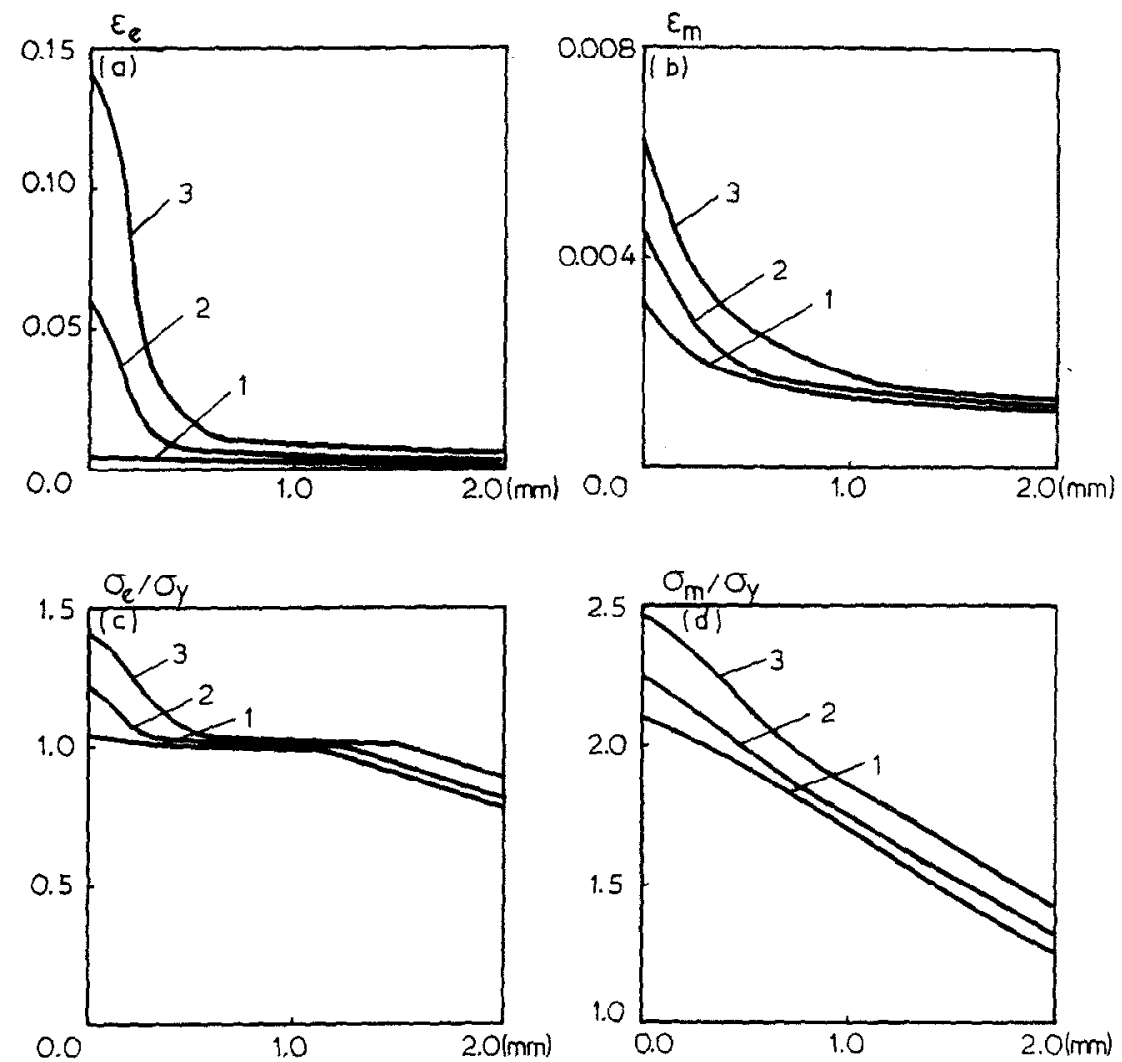

Fig. 5. The distributions of stress and strain near the crack tip in a three point bend beam.

\section{MACRO-RESPONSES OF THE SPECIMENS}

To justify the constitutive equations used for analysis and to check the precision of the finite element computations, we need to make a comparison between the computed results and the corresponding experimental curves.

The first type of specimen we used had a pre-crack cut in the middle of its span and was sharpened by fatigue loading. The concise dimensions of its contour are as given in Fig. 1 with a thickness of $20 \mathrm{~mm}$ to guarantee the plane strain condition. The other specimen was an axisymmetric bar notched by a V-shaped cut. The local radius at the sharp end of the cut is ahout $0.1 \mathrm{~mm}$.

The material used for the specimens was a low carbon low alloy steel. Its chemical composition $(\% \mathrm{wt})$ is: $0.21 \mathrm{C}, 0.25 \mathrm{Si}, 0.66 \mathrm{Mn}, 0.017 \mathrm{P}, 0.010 \mathrm{~S}$ and $1.01 \mathrm{Cr}$. The procedure for heat treatment was to have the material quenched from $880^{\circ} \mathrm{C}$ and tempered at $710^{\circ} \mathrm{C}$ for $5 \mathrm{~h}$. During testing, both types of specimens were loaded by an Instron-1195 test machine.

Figure 7 a shows the load-deflection curve of the three-point bend beam loaded at the centre by a concentrated force, while Fig. $7 \mathrm{~b}$ refers to the load-elongation curve of the axisymmetric bar notched by a V-shaped cut and loaded along its axial direction. The experimental curves are depicted by solid lines marked by $t$. Good accordance is seen between the computed results (lines marked by $c$ ) and those of the tests.

It should be emphasized that this sort of material has a flat plateau on its stress-strain curve (see Fig. 2a) and behaves in an ideally plastic manner after initial yielding until the equivalent strain $\varepsilon_{e}$ reaches about 0.02 . Afterwards, it is strain-hardened quickly. If we use the Ramberg-Osgood formula to characterize this material, there is no unique value for the hardening exponent that can be taken as appropriate. From Figs 5 and 6, we can see that, besides a small zone around the crack tip or notch tip, most of the plastic region is still in its small strain stage with equivalent strain less than 0.02 . However, in the strain concentrated zone the maximum value of strain can be larger than 0.2 in association with our mesh size. If a unique value of hardening exponent were used, the computed load response of the specimen would be much higher than those predicted in Fig. 7, 

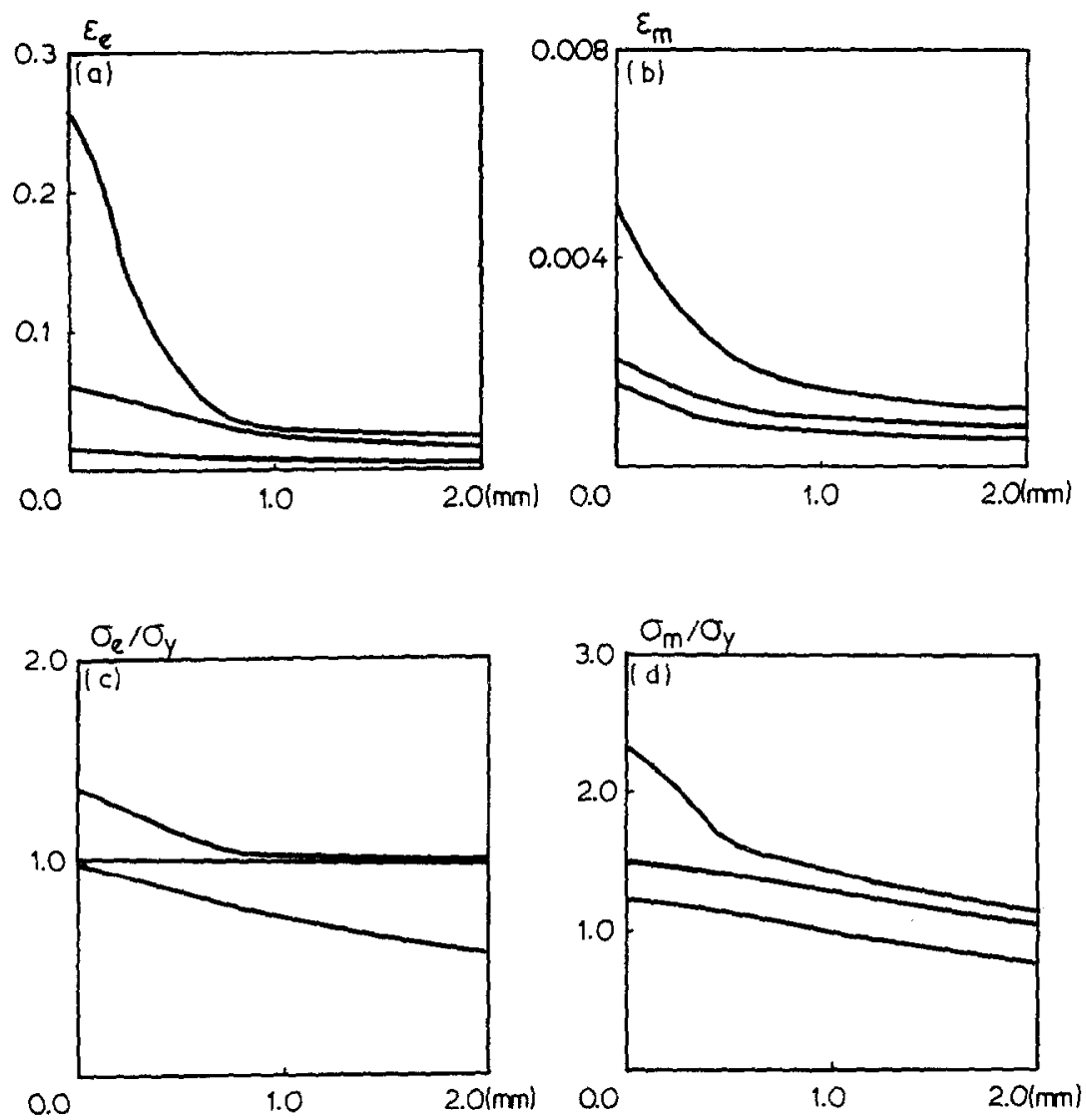

Fig. 6. The distributions of stress and strain near the notch tip in an axisymmetric bar.

because any hardening exponent chosen must yield a harder response than that of a (nearly) ideally plastic one.

\section{SINGULARITY IN A CRACK TIP FIELD}

Based on a deformation type of constitutive theory, Hutchinson [1] and Rice and Rosengren [2] found a new singular field different from the one characterized by the stress intensity factor in
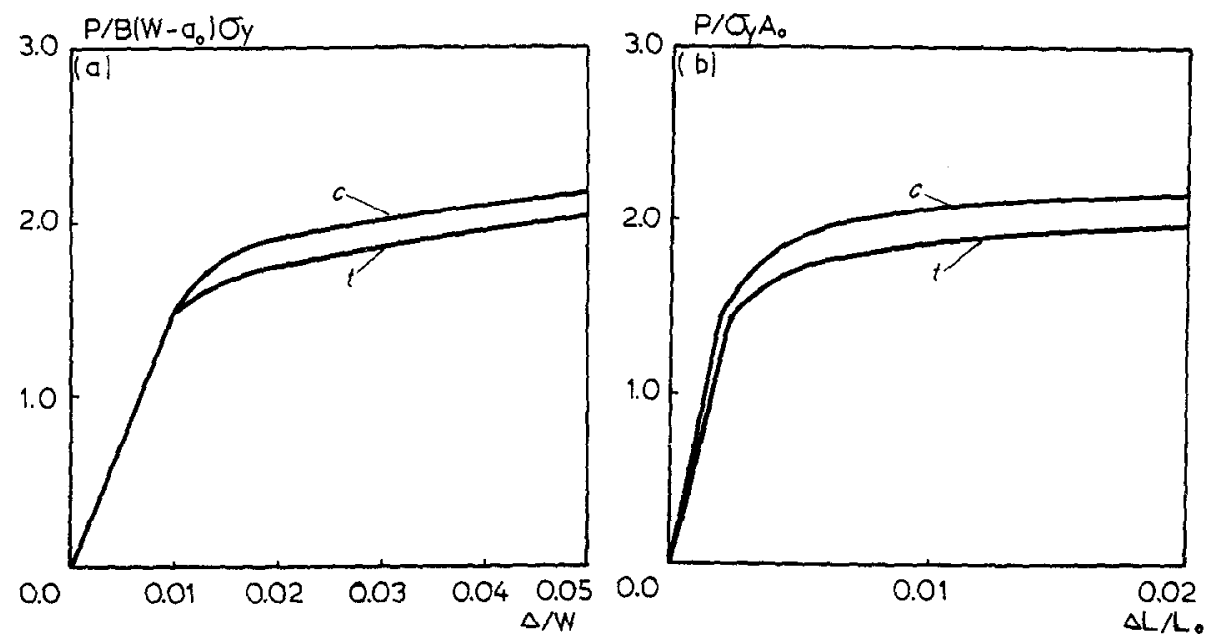

Fig. 7. The macroscopic responses in (a) a three-point bend beam and (b) an axisymmetric bar notched by a V-shaped cut. 
elasticity for the plastic zone near a crack tip. This is now known as the HRR field. With regard to this model, the equivalent stress distribution near the crack tip takes the form

$$
\sigma_{e} \propto K_{0}\left(\frac{1}{r}\right)^{1 / n+1}
$$

where $K_{0}$ is an amplitude factor related to the applied stress, $r$ denotes the distance from the crack tip and $n$ represents the hardening exponent of the material used.

Figure 8 shows the distribution of the equivalent stress $\sigma_{e}$ with respect to the distance $r$ from the crack tip border in the three-point bend beam.

Observing the stress and strain distributions approaching the crack tip shown in the log-log figure of $\sigma_{e}$ (normalized by $\sigma_{y}$ ) versus $r /$ CTOD in sequence, we find the following features:

(a) There is a straight line in the elastic zone, dominated by the stress intensity factor $K$.

(b) Next to this the field is controlled by the HRR model in plasticity. Since the equivalent strain $\varepsilon_{e}$ in this region is still small, it falls into the yielding-plateau part of the stress-strain curve in Fig. 2a. Hence an approximate value of the hardening exponent can be obtained as $n=30$, although it varies greatly once $\varepsilon_{e}>0.02$.

(c) Owing to the blunting of the crack tip, the HRR field for stress shifts away from the tip towards the elastic field as the local strain is enhanced, leaving a crack tip blunting field behind.

With regard to the strains shown by Figs 5 and 6, both the equivalent strain and the mean strain caused by plastic dilatation are accumulated and well concentrated. The interaction between the deviatoric and volumetric parts should not be neglected. Therefore, any non-dilatant constitutive description of the material in the blunting field would not be appropriate once the strain is large.

Following the procedure of loading, we can say that initially the $K$ field is dominant throughout the whole field, except in the small-scale yielding zone. Then the HRR field comes into effect before crack tip blunting, at the stage of $\Delta / W \leqslant 0.01$ (which is the turning point in Fig. 4b), and the plastic zone ahead of the crack tip is still limited. As the plastic zone enlarges, blunting of the crack tip occurs and the influential zone of the HRR field shifts apart. An interesting point worth noticing is that when we use the normalized distance $r /$ CTOD as abscissa in Fig. 8, all the stress distributions at different stages of loading coincide with each other and the HRR field holds with the zone given by $(4 \leqslant r /$ CTOD $\leqslant 11)$. This indicates that $r / C T O D$ is an important characteristic measuring scale to find the self-similarity in stress distribution and the blunting field has its effect only within a characteristic length scale of $r /$ CTOD $<4$. This conclusion is in accordance with the numerical result of McMeeking [9] using incompressible Prantdl-Reuss materials and an initial crack tip with a finitc root radius. As the load reaches $\Delta / W=0.05$, crack tip blunting becomes very large with CTOA $>27^{\circ}$, and the damaged field will replace the blunting zone to be the most dominant field near the crack tip. Concerning this topic, Li et al. [4] have already made a report.
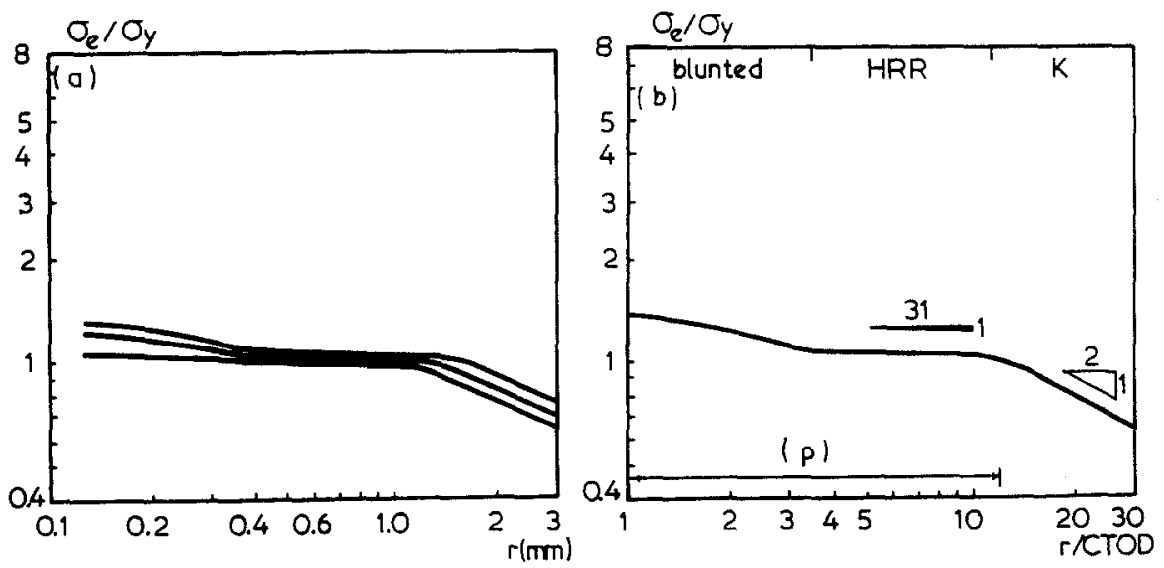

Fig. 8. The distributions of $\sigma_{e} / \sigma_{y}$ versus $r /$ CTOD. 


\section{CONCLUSIONS}

Based on the computational results given above, we can conclude the following.

1. Plastic dilatation is an important phenomena accompanied by the event of blunting at either the crack tip or notch tip. It is a stage of material behaviour, linking up that of small strain without damage and large strain including damage.

2. Blunting causes large local strains and a wide spread of plasticity or vice versa.

3. A self-similar distribution of stress near the crack tip is also found with respect to the parameter $r / C T O D$ with the material background of this paper.

4. As a consequence of blunting, the HRR field is shifted from the plane strain crack tip, $r / C T O D \geqslant 4$. That is, before the occurrence of local damage, blunting has its influential zone within $r /$ CTOD $<4$.

5. The good accordance obtained between the computed and tested curves of the macroscopic responses serves as a check for the constitutive equations used and for the precision of the computations undertaken.

Acknowledgements - This study was supported by the Laboratory for Nonlinear Mechanics of Continuous Media in the Institute of Mechanics, Academia Sinica.

\section{REFERENCES}

[1] J. W. Hutchinson, Singular behaviour at the end of a tensile crack in a hardening material. J. Mech. Phys. Solids 16, 13-31 (1968).

[2] J. R. Rice and G. F. Rosengren, Plane strain deformation near a crack tip in a power-law hardening material. J. Mech. Phys. Solids 16, 1-12 (1968).

[3] S. Aoki, K. Kishimoto, T. Yoshida and M. Sakata, A finite element study of the near crack tip deformation of a ductile material under mixed mode loading. J. Mech. Phys. Solids 35, 431-455 (1987).

[4] G. C. Li, H. Q. Liu, M. L. Du, Y. S. Hong and X. Zhang, Crack tip behaviour and crack propagation in ductile material. Fatigue Fracture Engng Mater. Struct. 15, 187-202 (1992).

[5] R. M. McMeeking and J. R. Rice, Finite-element formulations for problems of large elastic-plastic deformation. Int. J. Solids Structures 11, 601-616 (1975).

[6] G. C. Li and I. C. Howard, The effect of strain softening in the matrix material during void growth. J. Mech. Phys. Solids 31, 85-102 (1983).

[7] X. X. Xia, G. Y. Yang, Y. S. Hong and G. C. Li, Tests and analysis on the ductile fracture axisymmetric specimens. Mechanical Behaviour of Materials V, Proc. ICM 5, Beijing. China, Vol. 1, pp. 199-203 (1987).

[8] G. Rousselier, Finite deformation constitutive relations including ductile fracture damage. Proc. ITUAM Symp. on Three Dimensional Constitutive Relations and Ductile Fracture, Dourdan, France, pp. 331-355 (1981).

[9] R. M. McMeeking, Finite deformation analysis of crack-tip opening in elastic-plastic materials and implications for fracture. J. Mech. Phys. Solids 25, 257-381 (1977). 